\title{
THE QUEUE WITH IMPATIENCE: CONSTRUCTION OF THE STATIONARY WORKLOAD UNDER FIFO
}

\author{
PASCAL MOYAL, ${ }^{*}$ Université de Technologie de Compiègne
}

\begin{abstract}
In this paper we study the stability of queueing systems with impatient customers and a single server operating under a FIFO (first-in-first-out) discipline. We first give a sufficient condition for the existence of a stationary workload in the case of impatience until the beginning of service. We then provide a weaker condition of existence on an enriched probability space using the theory of Anantharam et al. (1997), (1999). The case of impatience until the end of service is also investigated.
\end{abstract}

Keywords: Stochastic recursion; stationary solution; queues with impatience; renovative event; enrichment of probability space

2010 Mathematics Subject Classification: Primary 60F17

Secondary 60K25; 60B 12

\section{Introduction}

In this paper we examine the stability of queueing systems with impatient customers: the customers agree to wait for service within a limited period of time. They abandon the system when their patience ends before they could reach the service booth. Such models are particularly adequate to describe operating systems under sharp delay requirements, such as multimedia and time sensitive telecommunication and computer networks, on-line audio/video traffic flows, call centres, or supply chains.

We explicitly construct a stationary state for these systems in the particular case of a single server obeying the FIFO (first-in-first-out) discipline. To this end, we study a stochastically recursive sequence representing the workload seen by an arriving customer. This sequence and its dynamics have been thoroughly studied in the GI/GI/1 case in [4] and [5]. In the G/G/1 context, the workload sequence is driven by a nonmonotonic recursive equation (see (7)); hence, a construction of Loynes's type, using a backwards recurrence scheme, is not possible. In this case we use the following more sophisticated techniques to construct a stationary workload.

(i) Borovkov's theory of renovating events (see [3, p. 115], [7], and [8]) provides a sufficient condition for the existence and uniqueness of a finite stationary workload. Under this condition, we can thus construct a stationary loss probability $\pi$, and provide bounds for $\pi$ (see (14)).

(ii) We provide a weaker condition for the existence of a stationary workload on the enriched probability space $\Omega \times \mathbb{R}_{+}$(where $\Omega$ is the Palm probability space of reference) using

Received 8 June 2009; revision received 16 February 2010.

* Postal address: Laboratoire de Mathématiques Appliquées de Compiègne, Université de Technologie de Compiègne, Département Génie Informatique, Centre de Recherches de Royallieu, BP 20 529, 60205 Compiegne Cedex, France.

Email address: pascal.moyal@utc.fr 
Anantharam and Konstantopoulos' construction (see [1] and [2]), which is based on tightness techniques.

In both cases, we use the fact that the workload sequence is strongly dominated by another sequence, which is driven by a monotonic recursive equation (see (2)). Then the coupling of the dominating sequence with a unique stationary state allows us to construct the stationary state of the dominated sequence.

We also address the case where the customers are impatient until the end of their service: they abandon the queue when their patience ends before a server could complete their service. This case turns out to be more simple, in the sense that the workload sequence is driven by a monotonic and continuous recursive random mapping. Then the stability problem can be handled using Loynes' scheme.

This paper is organized as follows. In Section 2 we make precise our basic assumptions and introduce the queue with impatience until the beginning of service. In Section 2.2 we address the problem of existence of construction points. We provide a sufficient condition for the existence and uniqueness of a stationary workload in Section 2.4, and for the existence on an enriched probability space in Section 2.6. In Section 3 we study the case of impatience until the end of service.

\section{The queue with impatient customers}

Let $\mathbb{R}, \mathbb{Z}, \mathbb{N}$, and $\mathbb{N}^{*}$ denote the sets of real numbers, integers, nonnegative integers, and positive integers, respectively. We consider a queue with impatient customers, $G / G / 1 / 1+G(b)$ (according to Barrer's notation; see [6]). On the probability space $(\Omega, \mathcal{F}, \mathrm{P})$, furnished with the measurable bijective flow $\left(\theta_{t}\right)_{t \in \mathbb{R}}$ under which $\mathrm{P}$ is stationary and ergodic, consider the $\theta_{t}$-compatible point process $N$, whose points $\left\{T_{n}\right\}_{n \in \mathbb{Z}}$ represent the arrivals of the customers $\left\{C_{n}\right\}_{n \in \mathbb{Z}}$, with the convention that $T_{0}$ is the last arrival before time $t=0$. The interarrivals are denoted by $\xi_{n}=T_{n+1}-T_{n}$ for all $n \in \mathbb{Z}$. The process $N$ is marked by the sequence $\left\{\sigma_{n}\right\}_{n \in \mathbb{Z}}$ of nonnegative random variables (RVs) representing the service durations requested by the customers. The queueing system has a single nonidling server, and its buffer is of infinite capacity. The customers are impatient until the beginning of their service, in that they leave the system if they do not reach the service booth before a given deadline. In other words, customer $C_{n}$ agrees to wait in line for a given period of time, say $D_{n}$ (his initial patience), and if the server is not available during this period, he leaves the system forever at time $T_{n}+D_{n}$. Here $\left\{D_{n}\right\}_{n \in \mathbb{Z}}$ is a sequence of nonnegative marks of $\left(N_{t}\right)_{t \in \mathbb{R}}$. It is furthermore assumed throughout that $\xi_{0}, \sigma_{0}$, and $D_{0}$ are integrable and that $\mathrm{P}\left[\xi_{0}>0\right]>0$. We denote by $\mathcal{X}_{t}$ the number of customers in the system (or congestion) at time $t$, for all $t \in \mathbb{R}$. We assume throughout that a customer cannot leave the system once he has entered the service booth, even if his deadline is reached during his service.

Let $\left(\Omega, \mathcal{F}, \mathrm{P}^{0}, \theta\right)$ be the Palm space of $N(\sigma, D)$, where $\theta:=\theta_{T_{1}}$ is the associated bijective discrete flow (we denote its measurable inverse by $\theta^{-1}$ ). Then, $\mathrm{P}^{0}$ is stationary and ergodic under $\theta$, i.e. for all $\mathcal{A} \in \mathcal{F}, \mathrm{P}^{0}\left[\theta^{-1} \mathcal{A}\right]=\mathrm{P}^{0}[\mathcal{A}]$ and all $\mathcal{A}$ that are $\theta$-invariant (i.e. such that $\theta \mathcal{A}=\mathcal{A}$ ) are of probability 0 or 1 . Note that, according to these axioms, all $\theta$-contracting events (such that $\mathrm{P}^{0}\left[\mathcal{A}^{\mathrm{c}} \cap \theta^{-1} \mathcal{A}\right]=0$ ) are of probability 0 or 1 . We define

$$
\theta^{n}=\theta \circ \theta \circ \cdots \circ \theta \text { and } \theta^{-n}=\theta^{-1} \circ \theta^{-1} \circ \cdots \circ \theta^{-1}
$$

for all $n \in \mathbb{N}$. Note that the sequence $\left\{\xi_{n}, \sigma_{n}, D_{n}\right\}_{n \in \mathbb{Z}}$ is stationary in that, for all $n$,

$$
\xi_{n}=\xi \circ \theta^{n}, \quad \sigma_{n}=\sigma \circ \theta^{n}, \quad \text { and } \quad D_{n}=D \circ \theta^{n},
$$


where $\xi:=\xi_{0}, \sigma:=\sigma_{0}$, and $D:=D_{0}$. Note moreover that the RVs $\xi, \sigma$, and $D$ are $\mathrm{P}^{0}$-integrable, and that $\mathrm{P}^{0}[\xi>0]>0$. We say that two sequences of RVs $\left\{X_{n}\right\}_{n \in \mathbb{N}}$ and $\left\{Y_{n}\right\}_{n \in \mathbb{N}}$ couple when there exists a $\mathrm{P}^{0}$-almost surely $\left(\mathrm{P}^{0}\right.$-a.s.) finite index $N$ such that they coincide for all $n \geq N$. We say that there is strong backwards coupling between $\left\{X_{n}\right\}_{n \in \mathbb{N}}$ and $\left\{Y \circ \theta^{n}\right\}_{n \in \mathbb{N}}$ provided that, for some $\mathrm{P}^{0}$-a.s. finite $\tau, X_{n} \circ \theta^{-n}=Y$ for any $n \geq \tau$.

As is well known (see, e.g. [3, p. 79]), the problems of existence and uniqueness of a stationary regime for random processes observed at the arrival times can be formulated in a simple manner on $\left(\Omega, \mathcal{F}, \mathrm{P}^{0}, \theta\right)$. Let $Y$ be an $\mathbb{R}$-valued $\mathrm{RV}$, and let $\phi$ be a random mapping $\mathbb{R} \mapsto \mathbb{R}$, for which we shall emphasize the randomness, when needed, by writing $\phi[\omega](x)$, the image of $x$ through $\phi$ for the sample $\omega$. The stochastic recursive sequence (SRS) initiated by $Y$ and driven by $\phi$ is defined by

$$
\begin{gathered}
X_{0}^{Y}(\omega)=Y(\omega), \quad \mathrm{P}^{0} \text {-a.s., } \\
X_{n+1}^{Y}(\omega)=\phi\left[\theta^{n} \omega\right]\left(X_{n}^{Y}(\omega)\right), \quad n \in \mathbb{N}, \mathrm{P}^{0} \text {-a.s. }
\end{gathered}
$$

Then, the problem of existence of a stationary regime for this sequence amounts to that of an $\mathrm{RV} Y$ such that $X_{n}^{Y}=Y \circ \theta^{n}$ for all $n \in \mathbb{N}$. This is in turn equivalent to saying that $Y$ solves the functional equation

$$
Y \circ \theta=\phi(Y), \quad \mathrm{P}^{0} \text {-a.s. }
$$

Throughout, the stability study of the queue with impatient customers will be handled under these settings, for several processes of interest.

\subsection{Preliminary result}

Let us define $x \vee y=\max (x, y), x \wedge y=\min (x, y)$, and $x^{+}=x \vee 0$ for any $x, y \in \mathbb{R}$. On $\left(\Omega, \mathcal{F}, \mathrm{P}^{0}, \theta\right)$, let $\alpha$ and $\beta$ be two integrable $\mathbb{R}_{+}$-valued $\mathrm{RVs}$ such that $\mathrm{P}^{0}[\beta>0]>0$. Let $F_{\alpha, \beta}$ be the real-valued random mapping defined by, for $x \in \mathbb{R}$,

$$
F_{\alpha, \beta}(x)=[x \vee \alpha-\beta]^{+} .
$$

The SRS $\left\{Y_{n}^{Z}\right\}_{n \in \mathbb{N}}$ initiated by $Z$ and driven by $F_{\alpha, \beta}$ is stationary if and only if $Z$ solves the equation

$$
Z \circ \theta=F_{\alpha, \beta}(Z) \text {. }
$$

We have the following result (which completes Lemma 5 of [11]).

Lemma 1. There exists a unique $\mathrm{P}^{0}$-a.s. finite solution $Y_{\alpha, \beta}$ of (2), given by

$$
Y_{\alpha, \beta}:=\left[\sup _{j \in \mathbb{N}^{*}}\left(\alpha \circ \theta^{-j}-\sum_{i=1}^{j} \beta \circ \theta^{-i}\right)\right]^{+} .
$$

Moreover, for any $\mathrm{P}^{0}$-a.s. finite and nonnegative $R V Z$, the sequence $\left\{Y_{n}^{Z}\right\}_{n \in \mathbb{N}}$ couples in the strong backwards sense with $\left\{Y_{\alpha, \beta} \circ \theta^{n}\right\}_{n \in \mathbb{N}}$, and there exists, $\mathrm{P}^{0}$-a.s., an infinity of indices such that $Y_{n}^{Z}=0$ if and only if

$$
\mathrm{P}^{0}\left[Y_{\alpha, \beta}=0\right]>0 .
$$

Proof. Equation (2) can be handled using Loynes's construction (see [3] and [10]) since the mapping $F_{\alpha, \beta}$ is $\mathrm{P}^{0}$-a.s. continuous and nondecreasing. Hence, $Y_{\alpha, \beta}$ classically reads as the $\mathrm{P}^{0}$-almost-sure limit of Loynes's sequence, defined by $\left\{Y_{n}^{0} \circ \theta^{-n}\right\}_{n \in \mathbb{N}}$. 
It is routine to check from Birkhoff's ergodic theorem (and the fact that $\beta$ is not identically 0 ) that $Y_{\alpha, \beta}$ is $\mathrm{P}^{0}$-a.s. finite. Finally, the coupling property follows from the fact that, for all nonnegative RVs $Z$ that are $\mathrm{P}^{0}$-a.s. finite (and, in particular, for $Z=Y_{\alpha, \beta}$ ),

$$
\left\{Y_{n}^{Z} \neq Y_{n}^{0} \text { for all } n \in \mathbb{N}\right\}=\left\{Y_{n}^{Z}=Z-\sum_{i=0}^{n-1} \beta \circ \theta^{i}>0 \text { for all } n \in \mathbb{N}\right\},
$$

which is of probability 0 from Birkhoff's theorem. The last statement is a classical consequence of this coupling property under ergodic assumptions.

\subsection{General case: construction points}

According to the assumptions made above, the total sojourn time of customer $C_{n}$ does not exceed $D_{n}+\sigma_{n}$, i.e. the sum of his initial patience and the time necessary for his service. On the other hand, it is at least equal to $\sigma_{n} \wedge D_{n}$, i.e. the time until he either loses patience and leaves the system or is immediately served. Hence, provided that $C_{n}$ entered the system before time $t\left(T_{n} \leq t\right)$, irrespective of whether he has already entered service or left the system before time $t$, his remaining maximal sojourn time at time $t$ (i.e. the length of time remaining before his originally latest possible departure time expires, if not already reached) is given by $\left[\sigma_{n}+D_{n}-\left(t-T_{n}\right)\right]^{+}$, whereas his remaining minimal sojourn time at time $t$ (i.e. the length of time remaining before his earliest possible departure time expires, if not already reached) is given by $\left[\sigma_{n} \wedge D_{n}-\left(t-T_{n}\right)\right]^{+}$. Hence, the largest remaining maximal sojourn time (LRMST) at time $t$ among all customers who entered before time $t$ is given by

$$
\mathcal{L}_{t}:=\max _{n=1, \ldots, N_{t}}\left[\sigma_{n}+D_{n}-\left(t-T_{n}\right)\right]^{+},
$$

and the largest remaining minimal sojourn time (LRmST) at time $t$, is given by

$$
\mathcal{M}_{t}:=\max _{n=1, \ldots, N_{t}}\left[\sigma_{n} \wedge D_{n}-\left(t-T_{n}\right)\right]^{+} .
$$

The two processes $\left(\mathcal{L}_{t}\right)_{t \in \mathbb{R}}$ and $\left(\mathcal{M}_{t}\right)_{t \in \mathbb{R}}$ clearly have càdlàg paths (i.e. paths that are continuous from the right with left limits). We define, for all finite nonnegative RVs $Y$ and $Z$ and all $n \in \mathbb{N}$, $L_{n}^{Y}:=\mathcal{L}_{T_{n}-}$ and $M_{n}^{Z}:=\mathcal{M}_{T_{n}-}$, the LRMST and LRmST just before the arrival of customer $C_{n}$, provided that $\mathcal{L}_{T_{0}-}=Y$ and $\mathcal{M}_{T_{0}-}=Z$, respectively. Then, it is easily checked that, $\mathrm{P}^{0}$-a.s. for all $n \in \mathbb{N}$,

$$
\begin{aligned}
L_{n+1}^{Z} & =\left[L_{n}^{Z} \vee\left(\sigma_{n}+D_{n}\right)-\xi_{n}\right]^{+}=F_{\sigma_{n}+D_{n}, \xi_{n}}\left(L_{n}\right), \\
M_{n+1}^{Z} & =\left[M_{n}^{Z} \vee\left(\sigma_{n} \wedge D_{n}\right)-\xi_{n}\right]^{+}=F_{\sigma_{n} \wedge D_{n}, \xi}\left(M_{n}\right),
\end{aligned}
$$

using the notation introduced in (1). Consequently, a stationary LRMST $L$ and a stationary LRmST $M$ satisfy

$$
L \circ \theta=F_{\sigma+D, \xi}(L), \quad M \circ \theta=F_{\sigma \wedge D, \xi}(M) .
$$

Consequently, in view of Lemma 1 , a unique couple $(L, M)$ exists, given by

$$
\begin{aligned}
& L=Y_{\sigma+D, \xi}=\left[\sup _{j \in \mathbb{N}^{*}}\left(\sigma_{-j}+D_{-j}-\sum_{i=1}^{j} \xi_{-i}\right)\right]^{+}, \\
& M=Y_{\sigma \wedge D, \xi}=\left[\sup _{j \in \mathbb{N}^{*}}\left(\sigma_{-j} \wedge D_{-j}-\sum_{i=1}^{j} \xi_{-i}\right)\right]^{+} .
\end{aligned}
$$


In particular, for any initial conditions $Y$ and $Z,\left\{L_{n}^{Y}\right\}_{n \in \mathbb{N}}$ and $\left\{M_{n}^{Z}\right\}_{n \in \mathbb{N}}$ couple respectively with $\left\{Y_{\sigma+D, \xi} \circ \theta^{n}\right\}_{n \in \mathbb{N}}$ and $\left\{Y_{\sigma \wedge D, \xi} \circ \theta^{n}\right\}_{n \in \mathbb{N}}$. Therefore, there are, P-a.s., an infinity of indices such that $L_{n}^{Y}=0$ if and only if $\mathrm{P}^{0}[L=0]>0$, and an infinity of indices such that $M_{n}^{Z}=0$ if and only if $\mathrm{P}^{0}[M=0]>0$. Now noting that, for all initial conditions and all $t \geq 0$,

$$
\left\{\mathcal{L}_{t}=0\right\} \subseteq\left\{\mathcal{X}_{t}=0\right\} \subseteq\left\{\mathcal{M}_{t}=0\right\},
$$

we have proven the following elementary result.

Theorem 1. The $G / G / s / s+G(b)$ queue empties $\mathrm{P}^{0}$-a.s. an infinite number of times if

$$
\mathrm{P}^{0}\left[\sup _{j \in \mathbb{N}^{*}}\left(\sigma_{-j}+D_{-j}-\sum_{i=1}^{j} \xi_{-i}\right) \leq 0\right]>0
$$

and only if

$$
\mathrm{P}^{0}\left[\sup _{j \in \mathbb{N}^{*}}\left(\sigma_{-j} \wedge D_{-j}-\sum_{i=1}^{j} \xi_{-i}\right) \leq 0\right]>0 .
$$

\subsection{The single-server FIFO queue: workload sequence}

Throughout Section 2, we assume that the single server obeys the FIFO discipline. For all $t \in \mathbb{R}$, denote by $\mathcal{W}_{t}$ the workload submitted to the server at time $t$, i.e. the quantity of work still to be achieved at this time, measured in time units. The process $\left(\mathcal{W}_{t}\right)_{t \in \mathbb{R}}$ has càdlàg paths, and we define, for all $n, W_{n}=W_{T_{n}^{-}}$. Its value at time $t$ equals the work generated by only those customers who arrive up to time $t$ and who will eventually be served, since the other customers will never reach the server. Under the FIFO discipline, the served customers are those whose patience upon arrival exceeds the workload $\mathcal{W}_{t}$. Between arrival times, the process $\left(\mathcal{W}_{t}\right)_{t \in \mathbb{R}}$ decreases at a unit rate. Hence, the workload sequence is driven by the recursive equation

$$
W_{n+1}=\left[W_{n}+\sigma_{n} \mathbf{1}_{\left\{W_{n} \leq D_{n}\right\}}-\xi_{n}\right]^{+} .
$$

In other words, the workload sequence is stochastically recursive, driven by the random map $\varphi$ defined by, for all $x \in \mathbb{R}$,

$$
\varphi(x)=\left[x+\sigma \mathbf{1}_{\{x \leq D\}}-\xi\right]^{+} .
$$

Hence, a stationary workload $W$ solves

$$
W \circ \theta=\varphi(W) .
$$

The random map $\varphi$ is not monotonic in the state variable; hence, a construction of Loynes's type is fruitless. In Section 2.4 we use renovating events to provide a sufficient condition for the existence and uniqueness of a solution to (7). In Section 2.6 we show that, under weaker assumptions, a solution exists on an enlarged probability space.

\subsection{Sufficient condition}

Theorem 2. If (5) holds, (7) admits a unique finite solution $W$ such that

$$
Y_{\sigma \wedge D, \xi} \leq W \leq Y_{\sigma+D, \xi}, \quad \mathrm{P}^{0}-a . s .
$$

where $Y_{\sigma+D, \xi}$ and $Y_{\sigma \wedge D, \xi}$ are defined by (3) and (4), respectively. Moreover, for any initial condition $Z$ such that $Z \leq Y_{\sigma+D, \xi}, \mathrm{P}^{0}$-a.s., there is strong backwards coupling for $\left\{W_{n}^{Z}\right\}_{n \in \mathbb{N}}$ with $\left\{W \circ \theta^{n}\right\}_{n \in \mathbb{N}}$. 
This result can be related to the classical stability result for the GI/GI/1/1+GI(b)-FIFO queue (i.e. all sequences are independent and identically distributed, and independent of one another); see [4, Lemma 2, p. 162] and also [5]. Hence, the recursive sequence $\left\{W_{n}\right\}_{n \in \mathbb{N}}$ is a Markov chain, which is proven to be ergodic whenever the generic patience $D$ is a.s. finite (an assumption that is made throughout this paper), and whenever

$$
\mathrm{P}[\sigma<\xi]>0 .
$$

The latter result follows from typical properties of Markov chains (Harris recurrence and irreducibility), and it is not surprising that our result, under more general assumptions, is weaker. More precisely, the existence of a stationary workload and the recurrence of 0 (Theorem 1) are entailed by Lemma 2 of [4] for a GI/GI/1/1+GI(b)-FIFO queue whenever (5) holds, and whenever either one of the $\mathrm{RVs} \xi$ and $\sigma$ is absolutely continuous, or $\mathrm{P}^{0}[D>0]=1$. Indeed, it then follows that on an event $\mathcal{A}$ such that $\mathrm{P}^{0}[\mathcal{A}]>0$,

$$
\sigma_{-1}-\xi_{-1}<\sigma_{-1}+D_{-1}-\xi_{-1} \leq 0
$$

and (8) holds in view of the stationarity of $\theta$.

Proof of Theorem 2. Existence. Let us first remark that, for any $x \in \mathbb{R}^{+}$,

$$
\begin{aligned}
\varphi(x) & =\left[x+\sigma \mathbf{1}_{\{x \leq D\}}+x \mathbf{1}_{\{D<x \leq D+\sigma\}}+x \mathbf{1}_{\{D+\sigma<x\}}-\xi\right]^{+} \\
& \leq\left[(D+\sigma) \mathbf{1}_{\{x \leq D\}}+(D+\sigma) \mathbf{1}_{\{D<x \leq D+\sigma\}}+x \mathbf{1}_{\{D+\sigma<x\}}-\xi\right]^{+} \\
& =[x \vee(\sigma+D)-\xi]^{+} \\
& =F_{\sigma+D, \xi}(x), \quad \mathrm{P}^{0} \text {-a.s. }
\end{aligned}
$$

In view of the almost-sure increasingness of $F_{\sigma+D, \xi}$, we have, in particular, for all $x \leq y$,

$$
\varphi(x) \leq F_{D+\sigma, \xi}(y), \quad \mathrm{P}^{0} \text {-a.s. }
$$

and a straightforward induction shows that $Z \leq Y_{\sigma+D, \xi}$ implies that

$$
W_{n}^{Z} \leq Y_{\sigma+D, \xi} \circ \theta^{n}, \quad n \geq 0 .
$$

Therefore, define the event

$$
\mathcal{A}_{n}:=\left\{Y_{\sigma+D, \xi} \circ \theta^{n}=0\right\} \text { for all } n .
$$

The sequence $\left\{\mathcal{A}_{n}\right\}_{n \in \mathbb{N}}$ is a sequence of renovating events of length 1 for $\left\{W_{n}^{Z}\right\}_{n \in \mathbb{N}}$ (see $[3$, p. 115], [7], and [8]), since

$$
\mathcal{A}_{n} \subseteq\left\{W_{n}^{Z}=0\right\}, \quad n \geq 0 .
$$

Moreover, this sequence is stationary in the sense that, for all $n \geq 0$,

$$
\mathcal{A}_{n}=\theta^{-n} \mathcal{A}_{0}, \quad n \geq 0 .
$$

Consequently, since (5) amounts to $\mathrm{P}^{0}\left[\mathcal{A}_{0}\right]>0$, this is, from [3, Theorem 2.5.3], a sufficient condition for the existence of a solution $W$ to (7), and for strong backwards coupling to occur for $\left\{W_{n}^{Z}\right\}_{n \in \mathbb{N}}$ with $W$. 
Uniqueness. Let $W$ be a solution of (7). First, assuming that $W>D, \mathrm{P}^{0}$-a.s. (which implies in particular that $W \circ \theta>0, \mathrm{P}^{0}$-a.s.) yields

$$
W \circ \theta=W-\xi, \quad \mathrm{P}^{0} \text {-a.s. }
$$

an absurdity in view of the ergodic lemma (see [3, Lemma 2.2.1]). Therefore, we have

$$
\mathrm{P}^{0}[W \leq D]>0
$$

On the other hand, in view of (10), on the event $\left\{W \leq Y_{\sigma+D, \xi}\right\}$,

$$
W \circ \theta=\varphi(W) \leq F_{\sigma+D, \xi}\left(Y_{\sigma+D, \xi}\right)=Y_{\sigma+D, \xi} \circ \theta .
$$

Thus, $\left\{W \leq Y_{\sigma+D, \xi}\right\}$ is $\theta$-contracting, whereas, on $\{W \leq D\}$,

$$
W \circ \theta=\left[(W+\sigma) \mathbf{1}_{\{W \leq D\}}-\xi\right]^{+} \leq\left[(D+\sigma) \mathbf{1}_{\{W \leq D\}}-\xi\right]^{+} \leq Y_{\sigma+D, \xi} \circ \theta,
$$

which shows in view of (11) that

$$
0<\mathrm{P}^{0}[W \leq D] \leq \mathrm{P}^{0}\left[W \circ \theta \leq Y_{\sigma+D, \xi} \circ \theta\right]=\mathrm{P}^{0}\left[W \leq Y_{\sigma+D, \xi}\right] .
$$

Therefore, the event $\left\{W \leq Y_{\sigma+D, \xi}\right\}$ is $\mathrm{P}^{0}$-almost sure. As a consequence, $\left\{W_{n}^{W}\right\}_{n \in \mathbb{N}}=$ $\left\{W \circ \theta^{n}\right\}_{n \in \mathbb{N}}$ admits $\left\{\mathcal{A}_{n}\right\}_{n \in \mathbb{N}}$ as a stationary sequence of renovating events of length 1 . From [3, Remark 2.5.3], $\mathrm{P}^{0}\left[\mathcal{A}_{0}\right]>0$ implies the uniqueness property.

Finally, for any $x \in \mathbb{R}_{+}$, we have, $\mathrm{P}^{0}$-a.s.,

$$
\begin{aligned}
F_{\sigma \wedge D, \xi}(x) & =\left[(\sigma \wedge D) \mathbf{1}_{\{x \leq D \wedge \sigma\}}+x \mathbf{1}_{\{x>D \wedge \sigma\}} \mathbf{1}_{\{x \leq D\}}+x \mathbf{1}_{\{x>D\}}-\xi\right]^{+} \\
& \leq\left[(x+\sigma) \mathbf{1}_{\{x \leq D \wedge \sigma\}} \mathbf{1}_{\{x \leq D\}}+(x+\sigma) \mathbf{1}_{\{x>D \wedge \sigma\}} \mathbf{1}_{\{x \leq D\}}+x \mathbf{1}_{\{x>D\}}-\xi\right]^{+} \\
& =\varphi(x) .
\end{aligned}
$$

In view of the almost-sure increasingness of $F_{\sigma \wedge D, \xi}$, this implies that, on the event $\left\{Y_{\sigma \wedge D, \xi} \leq\right.$ $W\}$,

$$
Y_{\sigma \wedge D, \xi} \circ \theta=F_{\sigma \wedge D, \xi}\left(Y_{\sigma \wedge D, \xi}\right) \leq \varphi(W)=W \circ \theta
$$

thus, $\left\{Y_{\sigma \wedge D, \xi} \leq W\right\}$ is $\theta$-contracting. It is $\mathrm{P}^{0}$-almost sure since it includes the event $\left\{Y_{\sigma+D, \xi}=\right.$ $0\}$ (in view of the immediate fact that $Y_{\sigma \wedge D, \xi} \leq Y_{\sigma+D, \xi}, \mathrm{P}^{0}$-a.s.).

As is customary, under the FIFO discipline, the construction of the stationary versions of several quantities of interest can be derived from that of the workload sequence. In particular, provided that (5) holds, we can construct a congestion process and a departure process that are jointly compatible with the arrival process $\left(N_{t}\right)_{t \in \mathbb{R}}$. Let us remark that, under condition (5), there also exists a stationary loss probability, denoted by $\pi(b)$, which is the probability that the waiting time proposed to a customer exceeds his initial patience, at equilibrium. This reads as

$$
\pi(b)=\mathrm{P}^{0}[W>D] .
$$

With Theorem 2 in hand, we have

$$
\mathrm{P}^{0}\left[Y_{\sigma \wedge D, \xi}>D\right] \leq \pi(b) \leq \mathrm{P}^{0}\left[Y_{\sigma+D, \xi}>D\right] .
$$




\subsection{Counterexamples}

Let us now focus on cases where condition (5) does not hold. As we show in the following counterexamples, it is then easy to construct examples where uniqueness and even existence are not granted when working on the original probability space. Let $\Omega:=\left\{\omega_{1}, \omega_{2}\right\}$. Denote by $\mathrm{P}^{0}$ the uniform probability on $\Omega$, and define the shift $\theta$ on $\left(\Omega, \mathrm{P}^{0}\right)$ by $\theta\left(\omega_{1}\right)=\omega_{2}$ and $\theta\left(\omega_{2}\right)=\omega_{1}$. Hence, $\theta$ is clearly stationary and ergodic under $\mathrm{P}^{0}$. Note that any solution $W$ of (7) satisfies

$$
\begin{aligned}
& W\left(\omega_{2}\right)=\left[W\left(\omega_{1}\right)+\sigma\left(\omega_{1}\right) \mathbf{1}_{\left\{W\left(\omega_{1}\right) \leq D\left(\omega_{1}\right)\right\}}-\xi\left(\omega_{1}\right)\right]^{+}, \\
& W\left(\omega_{1}\right)=\left[W\left(\omega_{2}\right)+\sigma\left(\omega_{2}\right) \mathbf{1}_{\left\{W\left(\omega_{2}\right) \leq D\left(\omega_{2}\right)\right\}}-\xi\left(\omega_{2}\right)\right]^{+} .
\end{aligned}
$$

Example 1. (Nonexistence.) Let the RVs $\xi, \sigma$, and $D$ be defined on $\left(\Omega, \mathrm{P}^{0}, \theta\right)$, and satisfy

$$
\begin{gathered}
\xi\left(\omega_{1}\right)=\xi\left(\omega_{2}\right)=1, \\
\sigma\left(\omega_{1}\right)>2, \quad \sigma\left(\omega_{2}\right) \neq 2, \\
D\left(\omega_{1}\right) \geq 3, \quad D\left(\omega_{2}\right) \leq 2 .
\end{gathered}
$$

First, if $W\left(\omega_{1}\right) \leq 3$ then in view of $(15)$,

$$
W\left(\omega_{2}\right)=\left[W\left(\omega_{1}\right)+\sigma\left(\omega_{1}\right)-1\right]^{+}=W\left(\omega_{1}\right)+\sigma\left(\omega_{1}\right)-1 .
$$

Then, with (16),

$$
\begin{aligned}
W\left(\omega_{1}\right) & =\left[W\left(\omega_{1}\right)+\sigma\left(\omega_{1}\right)-2+\sigma\left(\omega_{2}\right) \mathbf{1}_{\left\{W\left(\omega_{2}\right) \leq D\left(\omega_{2}\right)\right\}}\right]^{+} \\
& =W\left(\omega_{1}\right)+\sigma\left(\omega_{1}\right)-2+\sigma\left(\omega_{2}\right) \mathbf{1}_{\left\{W\left(\omega_{2}\right) \leq D\left(\omega_{2}\right)\right\}} \\
& >W\left(\omega_{1}\right)
\end{aligned}
$$

which is absurd. Hence, $W\left(\omega_{1}\right)>3$, but then $W\left(\omega_{2}\right)=\left[W\left(\omega_{1}\right)-1\right]^{+}=W\left(\omega_{1}\right)-1>2 \geq$ $D\left(\omega_{2}\right)$, which, with $(16)$, yields $W\left(\omega_{1}\right)=\left[W\left(\omega_{1}\right)-2\right]^{+}=W\left(\omega_{1}\right)-2$, another absurdity. There is no solution to (7).

Example 2. (Nonuniqueness.) Assume that

$$
\begin{gathered}
\xi\left(\omega_{1}\right)=\xi\left(\omega_{2}\right)=1, \\
\sigma\left(\omega_{1}\right) \in[0,2], \quad \sigma\left(\omega_{2}\right)=2-\sigma\left(\omega_{1}\right), \\
D\left(\omega_{1}\right)>\left(1-\sigma\left(\omega_{1}\right)\right)^{+}, \quad D\left(\omega_{2}\right) \leq D\left(\omega_{1}\right)+\sigma\left(\omega_{1}\right)-1 .
\end{gathered}
$$

Then, it is easily seen that, for any $x \in\left(\left(1-\sigma\left(\omega_{1}\right)\right)^{+}, D\left(\omega_{1}\right)\right]$, the RV defined by

$$
W\left(\omega_{1}\right)=x, \quad W\left(\omega_{2}\right)=x+\sigma\left(\omega_{1}\right)-1
$$

is a solution to (7).

\subsection{Weak stationarity}

In this section we show how the techniques developed in [1] allow us to construct a stationary workload for the queue under weaker assumptions, on a probability space that is enriched with respect to the original one. This is done again using the stochastic comparison with the LRMST sequence (see (9)). 
Throughout this section, we suppose without loss of generality that $(\Omega, \mathcal{F})$ is Polish (see [1] for a precise definition). We work on the enlarged probability space $\Omega \times \mathbb{R}$, on which we define the shift

$$
\tilde{\theta}(\omega, x)=(\theta \omega, \varphi[\omega](x)) .
$$

We say that a subset $\mathcal{I} \subset \mathbb{R}$ is locally finite if $\mathcal{I} \cap \mathcal{A}$ has a finite cardinal for any interval $\mathcal{A}$. The following result holds.

Theorem 3. Suppose that either (5) or the following condition holds.

(A) The RVs $\sigma$ and $\xi$ are valued in a common locally finite space $\mathcal{I}$ that includes 0 , and is closed under addition.

Then, the stochastic recursion (7) admits a weak solution in the sense of [1], that is, a $\tilde{\theta}$-invariant probability $\tilde{\mathrm{P}}^{0}$ on $\Omega \times \mathbb{R}$ whose $\Omega$-marginal is $\mathrm{P}^{0}$. Therefore, on $(\Omega \times \mathbb{R})$, there exists an $\mathbb{R} \times \mathcal{M}(\mathbb{R})$-valued $R V(\tilde{W}, \tilde{\varphi})$ satisfying

$$
\tilde{W} \circ \tilde{\theta}=\tilde{\varphi}(\tilde{W}), \quad \tilde{\mathrm{P}}^{0}-\text { a.s. }
$$

In particular, $\left\{(\tilde{W}, \tilde{\varphi}) \circ \tilde{\theta}^{n}\right\}_{n \in \mathbb{N}}$ is stationary under $\tilde{\mathrm{P}}^{0}$, and the $\Omega$-marginal of $\left\{\tilde{\varphi}\left[\tilde{\theta}^{n}(\cdot)\right]\right\}_{n \in \mathbb{N}}$ is the distribution of $\left\{\varphi\left[\theta^{n}(\cdot)\right]\right\}_{n \in \mathbb{N}}$.

Proof. We aim to apply Theorem 1 of [1], whose corrected version is presented in [2]. Let us check that its hypotheses are met in our case.

First, the sequence $\left\{L_{n}^{0}\right\}_{n \in \mathbb{N}}$ is tight since it converges weakly. On the other hand, (10) implies, using an immediate induction, that, $\mathrm{P}^{0}$-a.s., $W_{n}^{0} \leq L_{n}^{0}$ for all $n \in \mathbb{N}$. Hence, $\left\{W_{n}^{0}\right\}_{n \in \mathbb{N}}$ is tight, since, for all $\varepsilon>0$, there exists $M_{\varepsilon}$ such that, for all $n \in \mathbb{N}$,

$$
\mathrm{P}^{0}\left[W_{n}^{0} \leq M_{\varepsilon}\right] \geq \mathrm{P}^{0}\left[L_{n}^{0} \leq M_{\varepsilon}\right] \geq 1-\varepsilon .
$$

Now on $\Omega \times \mathbb{R}$ define the RVs

$$
\tilde{W}(\omega, x):=x, \quad \tilde{\varphi}[\omega, x]:=\varphi[\omega],
$$

and, for all $n \in \mathbb{N}$,

$$
\tilde{W}_{n}(\omega, x):=\tilde{W}\left(\tilde{\theta}^{n}(\omega, x)\right) .
$$

Note that, for all $n \in \mathbb{N}, \mathcal{A} \in \mathcal{F}$, and $\mathcal{B} \in \mathcal{B}(\mathbb{R})$,

$$
\mathrm{P}^{0} \otimes \delta_{0}\left[\tilde{\theta}^{-n}(\mathcal{A} \times \mathbb{R})\right]=\mathrm{P}^{0}\left[\theta^{-n} \mathcal{A}\right]=\mathrm{P}^{0}[\mathcal{A}]
$$

and

$$
\mathrm{P}^{0} \otimes \delta_{0}\left[\tilde{\theta}^{-n}(\Omega \times \mathscr{B})\right]=\mathrm{P}^{0} \otimes \delta_{0}\left[\left\{(\omega, x) \in \Omega \times \mathbb{R} ; W_{n}^{x}(\omega) \in \mathscr{B}\right\}\right]=\mathrm{P}^{0}\left[W_{n}^{0} \in \mathscr{B}\right] .
$$

Hence, the probability distributions $\left\{\left(\mathrm{P}^{0} \otimes \delta_{0}\right) \circ \tilde{\theta}^{-n}\right\}_{n \in \mathbb{N}}$ on $\Omega \times \mathbb{R}$ have $\Omega$-marginal $\mathrm{P}^{0}$ and $\mathbb{R}$ marginals, the distributions of $\left\{W_{n}^{0}\right\}_{n \in \mathbb{N}}$, which form a tight sequence. The sequence $\left\{\left(\mathrm{P}^{0} \otimes \delta_{0}\right) \circ \tilde{\theta}^{-n}\right\}_{n \in \mathbb{N}}$ is thus tight. Note that this entails in particular the tightness of the sequence

$$
\left\{\bar{Q}_{n}\right\}_{n \in \mathbb{N}}:=\left\{\frac{1}{n} \sum_{i=0}^{n-1}\left(\mathrm{P}^{0} \otimes \delta_{0}\right) \circ \tilde{\theta}^{-i}\right\}_{n \in \mathbb{N}}
$$




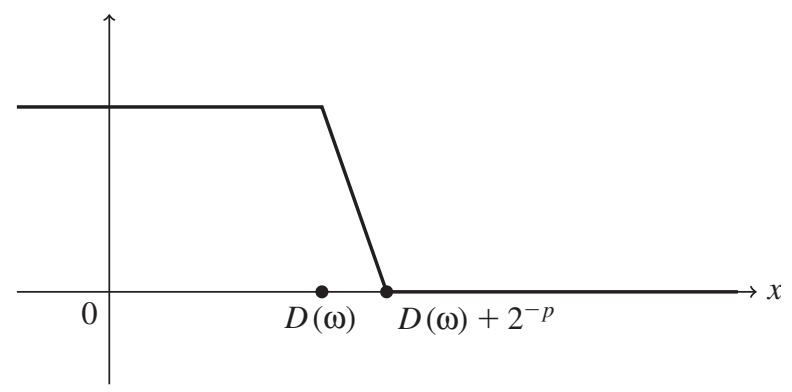

FiguRe 1: A path of $x \mapsto f_{p}(\omega, x)$.

We now aim to check condition (A3) of [2, p. 272]. To this end, let us define, for all $p \in \mathbb{N}^{*}$,

(i) $\mathcal{V}_{p}=\left\{(\omega, x) \in \Omega \times \mathbb{R} ; D(\omega)<x<D(\omega)+2^{-p}\right\}$,

(ii) for any $(\omega, x) \in \Omega \times \mathbb{R}$,

$$
f_{p}(\omega, x)=\mathbf{1}_{\{x \leq D(\omega)\}}+\left(-2^{p} x+1+2^{p} D(\omega)\right) \mathbf{1}_{\left\{(\omega, x) \in \mathcal{V}_{p}\right\}},
$$

from which a path is represented in Figure 1,

(iii) for any $(\omega, x) \in \Omega \times \mathbb{R}$,

$$
\tilde{\theta}_{p}(\omega, x)=\left(\theta \omega,\left[x+f_{p}(\omega, x) \sigma(\omega)-\xi(\omega)\right]^{+}\right) .
$$

It is then easily checked that, for all $p, \mathcal{V}_{p}$ is an open set, $\tilde{\theta}=\tilde{\theta}_{p}$ outside $\mathcal{V}_{p}$, and that $\tilde{\theta}_{p}$ is continuous from $\omega \times \mathbb{R}$ into itself.

Let us first assume that condition (A) holds. Note that, for any $i \geq 1, W_{i}^{0} \circ \theta^{-i}$ can be interpreted as the workload in the system at time 0 , assuming that customer $C_{-i}$ finds an empty system upon arrival. It is then easily checked by induction at the construction points (the instants at which a customer enters an empty system) that, for all $i \geq 1$,

$$
W_{i}^{0} \circ \theta^{-i} \in \mathcal{I}, \quad \mathrm{P}^{0} \text {-a.s. }
$$

Fix $p \geq 1$. As a consequence of (17), we have, for any $i \geq 1$,

$$
\begin{aligned}
& \mathrm{P}^{0}\left[W_{i}^{0} \circ \theta^{-i} \in\left(D, D+2^{-p}\right)\right] \\
& \quad \leq \mathrm{P}^{0}\left[\left\{\min \{\mathcal{I} \cap(D, D+1)\} \in\left(D, D+2^{-p}\right)\right\} \cap\{\mathcal{I} \cap(D, D+1) \neq \varnothing\}\right],
\end{aligned}
$$

and, therefore,

$$
\begin{aligned}
\lim _{p \rightarrow \infty} & \liminf _{n \rightarrow \infty} \bar{Q}_{n}\left(\mathcal{V}_{p}\right) \\
\quad & \lim _{p \rightarrow \infty} \liminf _{n \rightarrow \infty} \frac{1}{n} \sum_{i=1}^{n} \mathrm{P}^{0}\left[W_{i}^{0} \circ \theta^{-i} \in\left(D, D+2^{-p}\right)\right] \\
& \leq \lim _{p \rightarrow \infty} \mathrm{P}^{0}\left[\left\{\min \{\mathcal{I} \cap(D, D+1)\}-D \in\left(0,2^{-p}\right)\right\} \cap\{\mathcal{I} \cap(D, D+1) \neq \varnothing\}\right] \\
& =0 .
\end{aligned}
$$


All the same, whenever (5) holds, in view of Theorem 2, there exists, $\mathrm{P}^{0}$-a.s., a finite $\tau$ such that $W_{i}^{0} \circ \theta^{-i}=W$ for all $i \geq \tau$. Hence, the random set

$$
\mathcal{E}=\left\{W_{i}^{0} \circ \theta^{-i} ; i \geq 1\right\}=\left\{W_{i}^{0} \circ \theta^{-i} ; i \leq \tau\right\}
$$

is a.s. of finite cardinal, so that (18) holds, replacing $\mathcal{L}$ by $\mathcal{E}$.

In conclusion, assumption (A3) of [2, p. 272] is verified under both condition (A) and (5). Hence, Theorem 1 of [1] holds, meaning that there exists a $\tilde{\theta}$-invariant probability $\tilde{\mathrm{P}}^{0}$ on $\Omega \times \mathbb{R}$ whose $\Omega$-marginal is $\mathrm{P}^{0}$.

It is now straightforward to show that

$$
\tilde{W} \circ \tilde{\theta}(\omega, x)=\varphi[\omega](x)=\tilde{\varphi}[\omega, x](\tilde{W}(\omega, x)), \quad \tilde{\mathrm{P}}^{0} \text {-a.s. }
$$

so that, for all $n \in \mathbb{N}, \tilde{\mathrm{P}}^{0}$-a.s.,

$$
\tilde{W}_{n+1}=\tilde{\varphi}\left[\tilde{\theta}^{n}(\cdot)\right]\left(\tilde{W}_{n}\right),
$$

where $\left\{\tilde{W}_{n}, \tilde{\varphi}\left[\tilde{\theta}^{n}(\cdot)\right]\right\}_{n \in \mathbb{N}}$ is stationary under $\tilde{\mathrm{P}}^{0}$.

Note that condition (A) typically holds whenever $\sigma$ and $\xi$ are valued in a lattice $\{x n ; n \in \mathbb{N}\}$, where $x>0$.

On another hand, note that condition (A) does not entail (5): take $\sigma\left(\omega_{1}\right)=3, \sigma\left(\omega_{2}\right)=1$, $D\left(\omega_{1}\right)=3$, and $D\left(\omega_{2}\right)=2$ in the first example of Section 2.5. Then, condition (A) holds for $\mathcal{I}=\mathbb{N}$, whereas (5) is violated: there exists a weak stationary solution, but no stationary solution on the original probability space.

2.6.1. Loss system. Consider a loss system $\mathrm{G} / \mathrm{G} / 1 / 1$ : there is no buffer, so each customer is served if and only if he finds an empty system upon arrival. This corresponds to a $\mathrm{G} / \mathrm{G} / 1 / 1+\mathrm{G}(\mathrm{b})$ queue in which the generic patience $D$ is null $\mathrm{P}^{0}$-a.s. The sufficient condition for the existence of a stationary workload, constructed in [3, Section 2.6], naturally corresponds to (5), taking $D \equiv 0$.

A stationary workload for this queue always exists on $\Omega \times \mathbb{R}$, as readily follows from Theorem 3, since

$$
E \subset\left\{\left[\sigma_{-i}-\sum_{j=1}^{i} \xi_{-j}\right]^{+} ; i \geq 1\right\},
$$

which has, $\mathrm{P}^{0}$-a.s., a finite cardinal since the sequence $\left\{\sigma_{-i}-\sum_{j=1}^{i} \xi_{-j}\right\}_{i \geq 1}$ tends to $-\infty$, $\mathrm{P}^{0}$-a.s., in view of Birkhoff's theorem. This result was proved in a similar way in [2], whereas an explicit construction of a stationary workload on $\Omega \times \mathbb{N}$ was proposed in [9] and [13] (see also [12] for a generalization of this result to a dominated lattice-valued SRS).

\section{Impatience until the end of service}

Let us now consider a $\mathrm{G} / \mathrm{G} / \mathrm{s} / \mathrm{s}+\mathrm{G}(\mathrm{e})$ queue: the model is the same as that of the previous sections, except that the customers are now assumed to remain impatient until the end of their service. Indeed, they leave the system, and are eliminated forever, if their service is not completed before their deadline. Using the notation and assumptions of the previous section, customer $C_{n}$ is thus discarded when the total time he has to wait in the buffer and spend in the service booth is larger than his initial time credit $D_{n}$. We assume that the customers are unaware of their waiting time and deadline, and so wait in the system, and possibly enter service, as long as their deadline is not reached. 


\subsection{Construction points}

For any $n \in \mathbb{Z}$, the maximal sojourn time of $C_{n}$ in the system is given by $D_{n}$, whereas its minimal sojourn time is $\sigma_{n} \wedge D_{n}$. Then, the LRmST sequence is that of the system with impatience until the beginning of service, whereas the LRMST sequence $\left\{L_{n}\right\}_{n \in \mathbb{N}}$ is driven by the recursive equation

$$
L_{n+1}=\left[L_{n} \vee D_{n}-\xi_{n}\right]^{+}=F_{D_{n}, \xi_{n}}\left(L_{n}\right),
$$

and, in view of Lemma 1, the unique stationary LRMST reads

$$
Y_{D, \xi}=\left[\sup _{j \in \mathbb{N}^{*}}\left(D_{-j}-\sum_{i=1}^{j} \xi_{-i}\right)\right]^{+} .
$$

As in Theorem 1, we have the following result.

Theorem 4. The $G / G / s / s+G(b)$ queue empties, $\mathrm{P}^{0}$-a.s., an infinite number of times if

$$
\mathrm{P}^{0}\left[\sup _{j \in \mathbb{N}^{*}}\left(D_{-j}-\sum_{i=1}^{j} \xi_{-i}\right) \leq 0\right]>0,
$$

and only if (6) holds.

\subsection{Single-server FIFO queue}

Now suppose that the discipline is FIFO. The patience of a customer may expire while he is in service. Hence, such customers contribute to the workload, since some service is provided to them, but their service is not completed. More precisely, the quantity of work added to the workload $W_{n}$ upon the arrival of customer $C_{n}$ is given by

$$
\begin{cases}\sigma_{n} & \text { if } W_{n} \leq\left(D_{n}-\sigma_{n}\right)^{+}, \\ \sigma_{n}-\left(W_{n}+\sigma_{n}-D_{n}\right)=D_{n}-W_{n} & \text { if }\left(D_{n}-\sigma_{n}\right)^{+}<W_{n} \leq D_{n} \\ 0 & \text { if } W_{n}>D_{n}\end{cases}
$$

This can be reformulated in the compact form

$$
W_{n+1}=\left[W_{n}+\left(\sigma_{n}-\left(W_{n}+\sigma_{n}-D_{n}\right)^{+}\right)^{+}-\xi_{n}\right]^{+} .
$$

Therefore, a stationary workload is an $\mathbb{R}_{+}$-valued $\mathrm{RV} S$ that solves the equation

$$
S \circ \theta=\psi(S):=\left[S+\left(\sigma-(S+\sigma-D)^{+}\right)^{+}-\xi\right]^{+} .
$$

We have the following result.

Theorem 5. (i) Equation (20) admits a $\mathrm{P}^{0}$-a.s. finite solution $S$ such that

$$
Y_{\sigma \wedge D, \xi} \leq S \leq Y_{D, \xi}, \quad \mathrm{P}^{0} \text {-a.s. }
$$

(ii) If(19) holds, this solution is unique and, for any $R V Z$ such that $Z \leq Y_{D, \xi}, \mathrm{P}^{0}$-a.s., $\left\{W_{n}^{Z}\right\}_{n \in \mathbb{N}}$ converges with strong backwards coupling to $S$.

(iii) If, in addition, (5) holds then the unique solution is such that $S \leq W, \mathrm{P}^{0}$-a.s., where $W$ is the only solution of (7). 
Proof. (i) The random mapping $\psi$ is $\mathrm{P}^{0}$-a.s. nondecreasing and continuous, which can be easily checked. Hence, a minimal solution $S$ to (20) can be constructed using Loynes' theorem. Let us now remark that, for any $x$,

$$
\begin{aligned}
\psi(x) & =\left[((x+\sigma) \wedge D) \mathbf{1}_{\{x \leq D\}}+x \mathbf{1}_{\{x>D\}}-\xi\right]^{+} \\
& \leq\left[(x \vee D) \wedge\left(x+\sigma \mathbf{1}_{\{x \leq D\}}\right)-\xi\right]^{+} \\
& =\varphi(x) \wedge F_{D, \xi}(x), \quad \mathrm{P}^{0} \text {-a.s. }
\end{aligned}
$$

This clearly implies that the event $\left\{S \leq Y_{D, \xi}\right\}$ is $\theta$-contracting. On the other hand, $S$ is such that $\mathrm{P}^{0}[S \leq D]>0$, since the contrary would imply that $S \circ \theta=S-\xi, \mathrm{P}^{0}$-a.s., a contradiction to the ergodic lemma. But, on $\{S \leq D\}$,

$$
S \circ \theta=[((S+\sigma) \wedge D)-\xi]^{+} \leq\left[D \vee Y_{D, \xi}-\xi\right]^{+}=Y_{D, \xi} \circ \theta .
$$

Consequently, we have

$$
S \leq Y_{D, \xi}, \quad \mathrm{P}^{0} \text {-a.s. }
$$

Now, for any $x$, we also have, $\mathrm{P}^{0}$-a.s.,

$$
\begin{aligned}
F_{\sigma \wedge D, \xi}(x)= & {\left[(D \wedge \sigma) \mathbf{1}_{\{x \leq D \wedge \sigma\}} \mathbf{1}_{\{x \leq D\}}+x \mathbf{1}_{\{x>D \wedge \sigma\}} \mathbf{1}_{\{x \leq D\}}+x \mathbf{1}_{\{x>D\}}-\xi\right]^{+} } \\
\leq & {\left[(D \wedge(x+\sigma)) \mathbf{1}_{\{x \leq D \wedge \sigma\}} \mathbf{1}_{\{x \leq D\}}+(D \wedge(x+\sigma)) \mathbf{1}_{\{x>D \wedge \sigma\}} \mathbf{1}_{\{x \leq D\}}\right.} \\
& \left.+x \mathbf{1}_{\{x>D\}}-\xi\right]^{+} \\
= & \psi(x),
\end{aligned}
$$

which implies that the event $\left\{Y_{\sigma \wedge D, \xi} \leq S\right\}$ is $\theta$-contracting. Assuming that $Y_{\sigma \wedge D, \xi}>\sigma \wedge D$, $\mathrm{P}^{0}$-a.s., would again contradict the ergodic lemma. Thus, $\left\{Y_{\sigma \wedge D, \xi} \leq S\right\}$ is $\mathrm{P}^{0}$-almost sure since, on $\left\{Y_{\sigma \wedge D, \xi} \leq \sigma \wedge D\right\}$,

$$
Y_{\sigma \wedge D, \xi} \circ \theta=[\sigma \wedge D-\xi]^{+} \leq\left[((S+\sigma) \wedge D) \mathbf{1}_{\{S \leq D\}}+S \mathbf{1}_{\{S>D\}}-\xi\right]^{+}=S \circ \theta .
$$

(ii) For any solution $S^{\prime}$ of (20), $\left\{S^{\prime} \leq S\right\}$ is $\theta$-contracting. This event is thus $\mathrm{P}^{0}$-almost sure whenever (19) holds since it is included in $\left\{Y_{D, \xi}=0\right\}$. Hence, the uniqueness of the solution is entailed by the minimality of $S$.

On the other hand, (21) implies by a simple induction that

$$
W_{n}^{Z} \leq L_{n}^{Y_{D, \xi}}=Y_{D, \xi} \circ \theta^{n}, \quad n \in \mathbb{N},
$$

whenever $Z \leq Y_{D, \xi}$. Thus, for any RV $Z$ such that $Z \leq Y_{D, \xi}, \mathrm{P}^{0}$-a.s., the sequence $\left\{\left\{Y_{D, \xi} \circ\right.\right.$ $\left.\left.\theta^{n}=0\right\}\right\}_{n \in \mathbb{N}}$ is a sequence of renovating events of length 1 for $\left\{W_{n}^{0}\right\}_{n \in \mathbb{N}}$. The strong backwards coupling property then follows, as in the proof of Theorem 2.

(iii) The fact that $\psi$ is $\mathrm{P}^{0}$-a.s. nondecreasing implies, together with (21), that, on the event $\{S \leq W\}$

$$
S \circ \theta \leq\left[W+\left(\sigma-(W+\sigma-D)^{+}\right)^{+}-\xi\right]^{+} \leq W \circ \theta .
$$

Therefore, $\{S \leq W\}$ is $\theta$-contracting. This event is $\mathrm{P}^{0}$-almost sure whenever (5) holds since it includes $\left\{Y_{D+\sigma, \xi}=0\right\}$.

In Figure 2 we represent a sample path of the random functions $\varphi, \psi, F_{\sigma \wedge D}, F_{D}$, and $F_{\sigma+D}$ to illustrate a comparison between (9), (12), (21), and (22). Note in particular that $\psi$ is continuous, whereas $\varphi$ is not. 


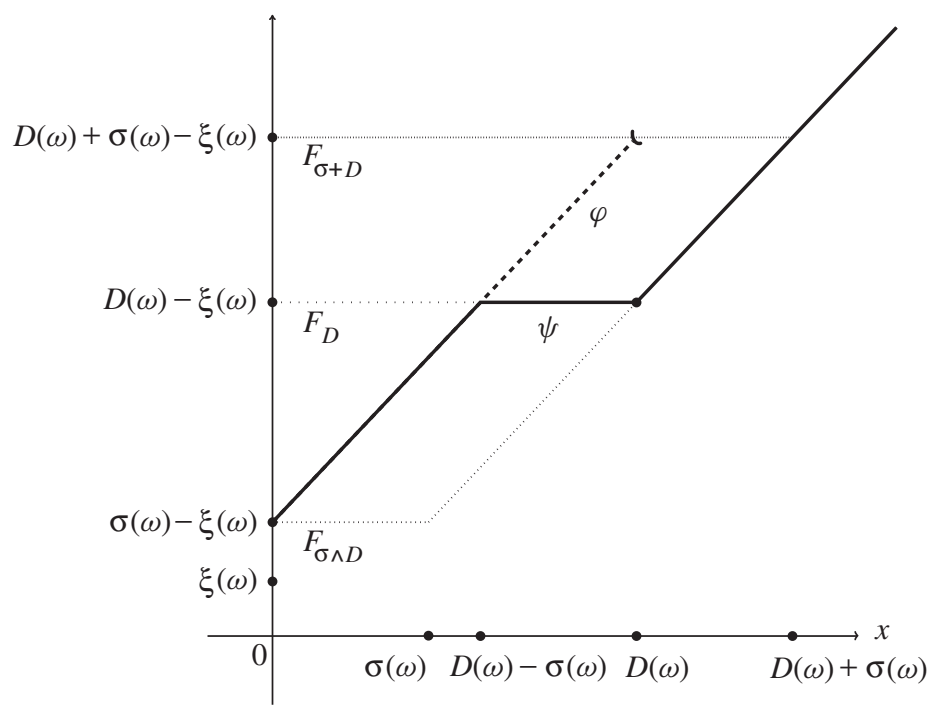

Figure 2: A sample path of $\varphi, \psi, F_{\sigma \wedge D}, F_{D}$, and $F_{\sigma+D}$.

For this model, the stationary loss probability $\pi(e)$ is the probability that the patience of customer $C_{0}$ is less than the sum of the stationary workload and his service time, i.e.

$$
\pi(e)=\mathrm{P}^{0}[S>D-\sigma] .
$$

From Theorem 5(i) we have

$$
\mathrm{P}^{0}\left[Y_{\sigma \wedge D, \xi}>D-\sigma\right] \leq \pi(e) \leq \mathrm{P}^{0}\left[Y_{D, \xi}>D-\sigma\right] .
$$

On the other hand, the stationary probability $\hat{\pi}(e)$ that a customer does not reach the server is given by

$$
\hat{\pi}(e)=\mathrm{P}^{0}[S>D] .
$$

Then in view of (13) and Theorem 5(ii), the loss probability $\pi(b)$ of the $\mathrm{G} / \mathrm{G} / 1 / 1+\mathrm{G}(\mathrm{b})$ queue is larger than $\hat{\pi}(e)$ for the same parameters.

\section{References}

[1] Anantharam, V. and Konstantopoulos, T. (1997). Stationary solutions of stochastic recursions describing discrete event systems. Stoch. Process. Appl. 68, 181-194.

[2] Anantharam, V. and Konstantopoulos, T. (1999). A correction and some additional remarks on: stationary solutions of stochastic recursions describing discrete event systems. Stoch. Process. Appl. 80, 271-278.

[3] Baccelli, F. ANd Brémaud, P. (2002). Elements of Queueing Theory, 2nd edn. Springer, Berlin.

[4] Baccelli, F. and Hébuterne, G. (1981). On queues with impatient customers. In Performance '81 (Amsterdam, 1981), North-Holland, Amsterdam, pp. 159-179.

[5] Baccelli, F., Boyer, P. and Hébuterne, G. (1984). Single-server queues with impatient customers. Adv. Appl. Prob. 16, 887-905.

[6] Barrer, D. Y. (1957). Queuing with impatient customers and ordered service. Operat. Res. 5, 650-656.

[7] Borovkov, A. A. (1976). Stochastic Processes in Queueing Theory. Springer, New York.

[8] Borovkov, A. A. AND Foss, S. G. (1992). Stochastically recursive sequences and their generalizations. Siberian Adv. Math. 2, 16-81.

[9] Flipo, D. (1983). Files d'attente à rejet à un serveur. Application au cas indépendant. C. R. Acad. Sci. Paris 297, 365-367. 
[10] Loynes, R. M. (1962). The stability of a queue with non-independent interarrival and service times. Proc. Camb. Phil. Soc. 58, 497-520.

[11] Moyal, P. (2008). Stability of a processor-sharing queue with varying throughput. J. Appl. Prob. 45, $953-962$.

[12] Moyal, P. (2009). Weak solutions of stochastic recursions: an explicit construction. Preprint. Available at http://arxiv.org/abs/0904.3240v1.

[13] Neveu, J. (1984). Construction de files d'attente stationnaires. In Modeling and Performance Evaluation Methodology (Lecture Notes Control Inf. Sci. 60), Springer, Berlin, pp. 29-41. 